\title{
On Complete Kähler Domains with $C^{1}$-Boundary
}

\author{
By \\ Takeo OHSAWA*
}

\begin{abstract}
Introduction
In the study of several complex variables the problem of characterizing Stein manifolds by their geometric propertics has been one of the central topics since Oka showed that every pseudoconvex unramified domain over the complex number space $\mathbb{C}^{n}$ is a Stein manifold. In 1956 Grauert [4] showed that every unramified domain over $\mathbb{C}^{n}$ with real analytic boundary and complete Kähler metric is a Stein manifold. He proved the pseudoconvexity of the domain and applied Oka's theorem. The purpose of the present note is to prove the following theorem.
\end{abstract}

Theorem: Let $X$ be a domain with $C^{1}$-boundary and with a complete Kähler metric in a complex manifold $M$. Then $X$ is pseudoconvex.

To prove the theorem we apply the method of $L^{2}$ estimates of $\bar{\partial}$ which is summarized in Section 1.

\section{§1. Differential Analysis on Complex Manifolds}

Let $X$ be a paracompact complex manifold of (complex) dimension $n$ and $d s^{2}$ be a hermitian metric on $X$. The complex vector space $C_{0}^{p, q}(X)$ of infinitely differentiable $(p, q)$ forms on $X$ with compact support has a structure of a pre-Hilbert space with respect to the norm $P$ defined by

$$
P(f):=\left(\int_{X} f \wedge \bar{*} f\right)^{1 / 2},
$$

where $f \in C_{0}^{p, q}(X)$ and $*$ denotes the conjugate of the star operator * associated to $d s^{2}$. We define

Received August 13, 1979.

* Research Institute for Mathematical Sciences, Kyoto University, Kyoto 606, Japan. 


$$
(f, g):=\int_{X} f \wedge \bar{*} g \quad \text { for } f, g \in C_{0}^{p, q}(X) .
$$

We introduce another norm $P_{\Phi}$ defined by

$$
P_{\Phi}(f):=\left(\int_{X} \exp (-\Phi) f \wedge \bar{*} f\right)^{1 / 2},
$$

where $f \in C_{0}^{p, q}(X)$ and $\Phi$ denotes a differentiable real valued function on $X$. We define

$$
(f, g)_{\Phi}:=\int_{X} \exp (-\Phi) f \wedge \bar{*} g, \quad \text { for } f, g \in C_{0}^{p, q}(X)
$$

We denote by $L_{1}^{p, q}(X)$ the square integrable $(p, q)$ forms on $X$, i.e., the completion of $C_{0}^{p, q}(X)$ with respect to the norm $P$, and denote by $L_{\Phi}^{p, q}(X)$ the completion of $C_{0}^{p, q}(X)$ with respect to $P_{\Phi}$. Note that if $\Phi$ is bounded on $X$, then the completion of $C_{0}^{p, q}(X)$ with respect to the norm $P_{\Phi}$ can be naturally identified with $L_{1}^{p, q}(X)$. A function $\Phi: X \rightarrow \mathbf{R}(\mathbf{R}=$ real numbers) is called strictly plurisubharmonic if $\Phi$ is $C^{2}$, i.e., twice continuously differentiable, and the Levi form

$$
\left(\frac{\partial^{2} \Phi}{\partial z^{\alpha} \partial \bar{z}^{\beta}}\right)
$$

is positive definite everywhere. Here $\left(z^{1}, \ldots, z^{n}\right)$ denotes a local coordinate system of $X$. Given a strictly plurisubharmonic function $\Phi$ on $X$,

$$
d \sigma^{2}:=\sum_{\alpha, \beta} \frac{\partial^{2} \Phi}{\partial z^{\alpha} \partial \bar{z}^{\beta}} d z^{\alpha} \cdot d \bar{z}^{\beta}
$$

defines a hermitian metric on $X$. Moreover the associated $(1,1)$ form

$$
\omega:=\sqrt{-1} \sum_{\alpha, \beta} \frac{\partial^{2} \Phi}{\partial z^{\alpha} \partial \bar{z}^{\beta}} d z^{\alpha} \wedge d \bar{z}^{\beta}
$$

is $d$-closed. As usual we say that a hermitian metric is a Kähler metric if the associated $(1,1)$ form is $d$-closed. Let $L_{2}^{p, q}(X)$ be the square integrable $(p, q)$ forms with respect to $d \sigma^{2}$, i.e., the completion of $C_{0}^{p, q}(X)$ with respect to the norm

$$
Q(f):=\left(\int_{X} f \wedge \bar{\Sigma} f\right)^{1 / 2}
$$

where $\bar{\tau}$ is the conjugate of the star operator $\bar{\tau}$ associated to $d \sigma^{2}$. Let $\left(\sigma_{1}, \ldots\right.$, $\sigma_{n}$ ) be a moving frame of the holomorphic cotangent bundle of $X$ which is orthonormal with respect to $d \sigma^{2}$ in a neighbourhood of $x_{0} \in X$. We let 


$$
d s^{2}=\sum_{i=1}^{n} \lambda_{i}^{2} d \sigma_{i} \cdot d \bar{\sigma}_{i},
$$

where $\lambda_{i}$ are positive valued functions in a neighbourhood of $x_{0}$ such that $\lambda_{1} \leqq \cdots \leqq \lambda_{n}$. Note that $\lambda_{i}$ are continuous functions and does not depend on the choice of $\left(\sigma_{1}, \ldots, \sigma_{n}\right)$, therefore $\lambda_{i}$ are well defined on $X$.

Proposition 1.1. If $\lambda_{i} \geqq 1$ for $i=1, \ldots, n$, then

$$
L_{2}^{n, q}(X) \subset L_{1}^{n, q}(X) \quad q \geqq 0
$$

and

$$
L_{2}^{p, 0}(X) \supset L_{1}^{p, 0}(X) \quad p \geqq 0 .
$$

In particular $L_{2}^{n, 0}(X)=L_{1}^{n, 0}(X)$.

Proof. For the proof of $L_{2}^{n, q}(X) \subset L_{1}^{n, q}(X)$ it is sufficient to show that

$$
Q(f) \geqq P(f), \quad \text { for } \quad f \in C_{0}^{n, q}(X) .
$$

Let us compute $f \wedge \bar{\xi} f$ at $x_{0}$. Let $\left(s_{1}, \ldots, s_{n}\right)$ and $\left(\sigma_{1}, \ldots, \sigma_{n}\right)$ be orthonormal moving frames of the holomorphic cotangent bundle of $X$ around $x_{0}$ with respect to $d s^{2}$ and $d \sigma^{2}$ respectively, such that

$$
s_{i}=\lambda_{i} \sigma_{i}
$$

Letting

$$
f=\sum_{i_{1}<\cdots<i_{q}} f_{i_{1} \cdots i_{q}} \sigma_{1} \wedge \cdots \wedge \sigma_{n} \wedge \bar{\sigma}_{i_{1}} \wedge \cdots \wedge \bar{\sigma}_{i_{q}}
$$

we have

$$
f \wedge \bar{\xi} f=K \sum_{i_{1}<\cdots<i_{q}}\left|f_{i_{1} \cdots i_{q}}\right|^{2} \sigma_{1} \wedge \cdots \wedge \sigma_{n} \wedge \bar{\sigma}_{1} \wedge \cdots \wedge \bar{\sigma}_{n},
$$

where

$$
K=(-1)^{n(n-1) / 2} 2^{-q}(\sqrt{-1})^{n} .
$$

On the other hand, since

$$
f=\sum_{i_{1}<\cdots<i_{q}} f_{i_{1} \cdots i_{q}} \frac{1}{\lambda_{1} \cdots \lambda_{n} \lambda_{i_{1}} \cdots \lambda_{i_{q}}} s_{1} \wedge \cdots \wedge s_{n} \wedge \bar{s}_{i_{1}} \wedge \cdots \wedge \bar{s}_{i_{q}}
$$

we have

$$
\begin{aligned}
f \wedge \bar{*} f & =K \sum_{i_{1}<\cdots<i_{q}}\left|f_{i_{1} \cdots i_{q}}\right|^{2} \frac{s_{1} \wedge \cdots \wedge s_{n} \wedge \bar{s}_{1} \wedge \cdots \wedge \bar{s}_{n}}{\lambda_{1}^{2} \cdots \lambda_{n}^{2} \lambda_{i_{1}}^{2} \cdots \lambda_{i_{q}}^{2}} \\
& =K \sum_{i_{1}<\cdots<i_{q}}\left|f_{i_{1} \cdots i_{q}}\right|^{2} \frac{\sigma_{1} \wedge \cdots \wedge \sigma_{n} \wedge \bar{\sigma}_{1} \wedge \cdots \wedge \bar{\sigma}_{n}}{\lambda_{i_{1}}^{2} \cdots \lambda_{i_{q}}^{2}}
\end{aligned}
$$


Hence we obtain $Q(f) \geqq P(f)$. The proof of $L_{2}^{p, 0}(X) \supset L_{1}^{p, 0}(X)$ is similar.

We define a first order differential operator

$$
\bar{\partial}: C_{0}^{p, q}(X) \rightarrow C_{0}^{p, q+1}(X)
$$

as follows;

$$
\begin{aligned}
\bar{\partial} f=(-1)^{p} \sum_{\substack{i_{1}<\cdots<i_{p} \\
j_{1}<\cdots<j_{q}}} \sum_{1 \leqq j \leqq n} \frac{\partial f_{i_{1} \cdots i_{p} \bar{j}_{1} \cdots \bar{j}_{q}}}{\partial \bar{z}^{j}} d z^{i_{1}} \wedge \cdots \wedge d z^{i_{p}} \wedge \\
d \bar{z}^{j} \wedge d \bar{z}^{j_{1}} \wedge \cdots \wedge d \bar{z}^{j_{q}},
\end{aligned}
$$

where

$$
f=\sum_{\substack{i_{1}<\cdots<i_{p} \\ j_{1}<\cdots<j_{q}}} f_{i_{1} \cdots i_{p} \bar{j}_{1} \cdots \bar{j}_{q}} d z^{i_{1}} \wedge \cdots \wedge d z^{i_{p}} \wedge d \bar{z}^{j_{1}} \wedge \cdots \wedge d \bar{z}^{j_{q}}
$$

The domain of $\bar{\partial}$ which we denote by $D^{p, q}$ is defined as the set of $f \in L_{1}^{p, q}(X)$ such that the current $\bar{\partial} f$ belongs to $L_{1}^{p, q+1}(X)$. We regard $\bar{\partial}$ as a closed linear operator from $L_{1}^{p, q}(X)$ to $L_{1}^{p, q+1}(X)$ with dense domain $D^{p, q}$. Since $D^{p, q}$ is dense in $L_{1}^{p, q}(X)$ the adjoint $\bar{\partial}^{*}$ of $\bar{\partial}$ is well defined in the following sense. $f$ belongs to the domain $D_{*}^{p, q+1}$ of $\bar{\partial}^{*}$ if and only if $f \in L_{1}^{p, q}(X)$ and there exists $h \in L_{1}^{p, q-1}(X)$ such that

$$
(h, u)=(f, \bar{\partial} u) \quad \text { for any } \quad u \in D^{p, q-1} .
$$

Such $h$ is uniquely determined by $f$ and we define $\bar{\partial}^{*} f=h$.

Note that $C_{0}^{p, q}(X) \subset D_{*}^{p, q}$ by the theorem of Stokes.

We define the adjoint of $\bar{\partial}$ with respect to the norm $P_{\Phi}$ similarly and denote it by $\bar{\partial}_{\Phi}^{*}$. The domain of $\bar{\partial}$ in $L_{\Phi}^{p, q}$ and the domain of $\bar{\partial}_{\Phi}^{*}$ is denoted by $D_{\Phi}^{p, q}$ and $D_{*}^{p, q}$, respectively.

We say that a hermitian metric $d s^{2}$ is complete if for any sequence $\left\{x_{v}\right\}$, $v=1,2, \ldots$ of points in $X$ which does not have an accumulation point, the distance between $x_{1}$ and $x_{v}$ tends to infinity as $v \rightarrow \infty$.

Proposition 1.2. Let $d s^{2}$ be a complete $C^{2}$ hermitian metric on $X$ and $\Phi$ be a $C^{2}$ function on $X$ with values in $\mathbf{R}$. Then $C_{0}^{p, q}(X)$ is dense in $D_{\Phi}^{p, q}, D_{*}^{p, q}$, and $D_{\Phi}^{p, q} \cap D_{*}^{p, q}$, with respect to the graph norms $P_{\Phi}(f)+P_{\Phi}(\bar{\partial} f), P_{\Phi}(f)+$ $P_{\Phi}\left(\bar{\partial}_{\Phi}^{*} f\right)$, and $P_{\Phi}(f)+P_{\Phi}(\bar{\partial} f)+P_{\Phi}\left(\bar{\partial}_{\Phi}^{*} f\right)$, respectively.

Proof. See Andreotti-Vesentini [1], Proposition 5.

Since $\bar{\partial}$ and $\bar{\partial}_{\phi}^{*}$ are closed operators we obtain 
Corollary 1.3. Under the same assumptions as above, $D_{\Phi}^{p, q}, D_{* \Phi}^{p, q}$, and $D_{\Phi}^{p, q} \cap D_{*}^{p, q}$ are the completions of $C_{0}^{p, q}(X)$ with respect to the graph norms.

Corollary 1.3 is not used in this paper.

From now on we assume that $X$ has a complete Kähler metric $d s^{2}$ of class $C^{2}$, there exists a bounded strictly plurisubharmonic function $\Phi$ on $X$, and $\lambda_{i}$ defined above with respect to $d s^{2}$ and $\Phi$ are $\geqq 1$. Then $P$ and $P_{\Phi}$ are epuivalent norms on $C_{0}^{p, q}(X)$ so that the completions of $C_{0}^{p, q}(X)$ with respect to $P$ and $P_{\Phi}$ agree.

Proposition 1.4. Let $X, d s^{2}$, and $\Phi$ be as above. Then we have the following inequality:

$$
C_{f}\left\{P_{\Phi}(\bar{\partial} u)+P_{\Phi}\left(\bar{\partial}_{\Phi}^{*} u\right)\right\} \geqq\left|(f, u)_{\Phi}\right|^{2} .
$$

Here $f \in L_{2}^{n, q}(X), q \geqq 1, u \in D_{\Phi}^{n, q} \cap D_{* \Phi}^{n, q}$, and $C_{f}$ is a continuous function of $Q(f)$.

Proof. In virtue of Proposition 1.2, it is sufficient to show ( $\dagger$ ) assuming $f, u \in C_{0}^{n, q}(X)$. Let $\Lambda$ be the operator defined by

$$
(\Omega \wedge u, v)=(u, \Lambda v),
$$

where $u \in C_{0}^{p, q}(X), v \in C_{0}^{p+1, q+1}(X)$ and $\Omega$ denotes the $(1,1)$ form associated to $d s^{2}$. The following formula is first due to Calabi-Vesentini [2];

$$
\left(\bar{\partial} \bar{\partial}_{\Phi}^{*}+\bar{\partial}_{\Phi}^{*} \bar{\partial}\right) u-*^{-1}\left(\bar{\partial} \bar{\partial}_{\Phi}^{*}+\bar{\partial}_{\Phi}^{*} \bar{\partial}\right) * u=(\Theta \Lambda-\Lambda \Theta) u \quad \text { for } \quad u \in C_{0}^{p, q}(X)
$$

where $\Theta$ denotes the left multiplication by $\omega$. From this equality it follows that

$$
P_{\Phi}(\bar{\partial} u)+P_{\Phi}\left(\bar{\partial}_{\Phi}^{*} u\right) \geqq(\Theta \Lambda u, u)_{\Phi} \quad \text { for } \quad u \in C_{0}^{n, q}(X) .
$$

So it remains to show that

$$
C_{f}(\Theta \Lambda u, u)_{\Phi} \geqq\left|(f, u)_{\Phi}\right|^{2} \quad \text { for } f, u \in C_{0}^{n, q}(X) \quad q \geqq 1 .
$$

For this purpose it is enough to show that

$$
q\left|(f, u)_{\Phi}\right|^{2} \leqq\left(\Lambda^{-1} \Theta^{-1} f, f\right)_{\Phi}(\Theta \Lambda u, u)_{\Phi}
$$

and

$$
\left(\Lambda^{-1} \Theta^{-1} f, f\right)_{\Phi} \leqq q C Q(f)^{2}
$$

for $f, u \in C_{0}^{n, q}(X), q \geqq 1$, where $C:=\sup _{x \in X} \exp (-\Phi(x))$ and we denote by $\Theta^{-1}$ and $\Lambda^{-1}$ the inverses of $\Theta: C_{0}^{n-1, q-1}(X) \rightarrow C_{0}^{n, q}(X)$ and $\Lambda: C_{0}^{n, q}(X) \rightarrow C_{0}^{n-1, q-1}(X)$, respectively. 
First we prove (21). Letting

$$
f=\sum_{i_{1}<\cdots<i_{q}} f_{i_{1} \cdots i_{q}} \sigma_{1} \wedge \cdots \wedge \sigma_{n} \wedge \bar{\sigma}_{i_{1}} \wedge \cdots \wedge \bar{\sigma}_{i_{q}}
$$

we have

$$
\Lambda^{-1} \Theta^{-1} f=\sum_{i_{1}<\cdots<i_{q}}\left(\sum_{k=1}^{q} \lambda_{i_{k}}^{2}\right) f_{i_{1} \cdots i_{q}} \sigma_{1} \wedge \cdots \wedge \sigma_{n} \wedge \bar{\sigma}_{i_{1}} \wedge \cdots \wedge \bar{\sigma}_{i_{q}}
$$

and

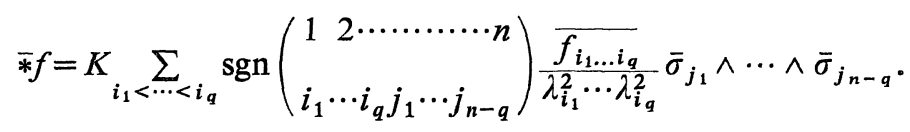

Thus we have

$$
\begin{aligned}
& \exp (-\Phi) \Lambda^{-1} \Theta^{-1} f \wedge \bar{*} f \\
& =K \exp (-\Phi) \sum_{i_{1}<\cdots<i_{q}} \frac{\sum_{k=1}^{q} \lambda_{i_{k}}^{2}}{\lambda_{i_{1}}^{2} \cdots \lambda_{i_{q}}^{2}}\left|f_{i_{1} \cdots i_{q}}\right|^{2} \sigma_{1} \wedge \cdots \wedge \sigma_{n} \wedge \bar{\sigma}_{1} \wedge \cdots \wedge \bar{\sigma}_{n} \\
& \leqq q K \exp (-\Phi) \sum_{i_{1}<\cdots<i_{q}}\left|f_{i_{1} \cdots i_{q}}\right|^{2} \sigma_{1} \wedge \cdots \wedge \sigma_{n} \wedge \bar{\sigma}_{1} \wedge \cdots \wedge \bar{\sigma}_{n} \\
& =q \exp (-\Phi) f \wedge \bar{\xi} f .
\end{aligned}
$$

Therefore

$$
\left(\Lambda^{-1} \Theta^{-1} f, f\right)_{\Phi} \leqq q C \int_{X} f \wedge \bar{\xi} f=q C Q(f)^{2} .
$$

Now we prove (20). In the proof of (21) we showed that

$$
\begin{aligned}
& \Lambda^{-1} \Theta^{-1} f=\sum_{i_{1}<\cdots<i_{q}}\left(\sum_{k=1}^{q} \lambda_{i_{k}}^{2}\right) f_{i_{1} \cdots i_{q}} \sigma_{1} \wedge \cdots \wedge \sigma_{n} \wedge \bar{\sigma}_{i_{1}} \wedge \cdots \wedge \bar{\sigma}_{i_{q}} \\
& =\sum_{i_{1}<\cdots<i_{q}}\left(\sum_{k=1}^{q} \frac{\lambda_{i_{k}}^{2}}{\lambda_{1} \cdots \lambda_{n} \lambda_{i_{1}} \cdots \lambda_{i_{q}}}\right) f_{i_{1} \ldots i_{k}} s_{1} \wedge \cdots \wedge s_{n} \wedge \bar{s}_{i_{1}} \wedge \cdots \wedge \bar{s}_{i_{q}}
\end{aligned}
$$

and

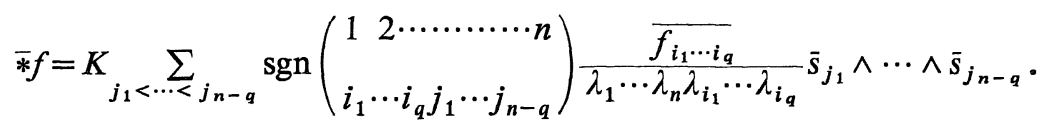

Hence if we let

$$
\begin{aligned}
& \tilde{f}=\sum_{i_{1}<\cdots<i_{q}} \tilde{f}_{i_{1} \cdots i_{q}} s_{1} \wedge \cdots \wedge s_{n} \wedge \bar{s}_{i_{1}} \wedge \cdots \wedge \bar{s}_{i_{q}} \\
& :=\sum_{i_{1}<\cdots<i_{q}} \sqrt{\sum_{k=1}^{q} \lambda_{i_{k}}^{2}} \frac{f_{i_{1} \cdots i_{q}}}{\lambda_{1} \cdots \lambda_{n} \lambda_{i_{1}} \cdots \lambda_{i_{q}}} s_{i} \wedge \cdots \wedge s_{n} \wedge \bar{s}_{i_{1}} \wedge \cdots \wedge \bar{s}_{i_{q}}
\end{aligned}
$$

we have

$$
\left(\Lambda^{-1} \Theta^{-1} f, f\right)_{\Phi}=(\tilde{f}, \tilde{f})_{\Phi}
$$


As for $u=\Sigma u_{i_{1} \cdots i_{q}} s_{1} \wedge \cdots \wedge s_{n} \wedge \bar{s}_{i_{1}} \wedge \cdots \wedge \bar{s}_{i_{q}}$ we have

$$
\Theta \Lambda u=\sum_{i_{1}<\cdots<i_{q}} \sum_{k=1}^{q} \frac{u_{i_{1} \cdots i_{q}}}{\lambda_{i_{k}}^{2}} s_{1} \wedge \cdots \wedge s_{n} \wedge \bar{s}_{i_{1}} \wedge \cdots \wedge \bar{s}_{,}
$$

Hence if we let

$$
\begin{aligned}
\tilde{u} & =\sum_{i_{1}<\cdots<i_{q}} \tilde{u}_{i_{1} \cdots i_{q}} s_{1} \wedge \cdots \wedge s_{n} \wedge \bar{s}_{i_{1}} \wedge \cdots \wedge \bar{s}_{i_{q}} \\
& :=\sum_{i_{1}<\cdots<i_{q}} \sqrt{\sum_{k=1}^{q} \frac{1}{\lambda_{i_{k}}^{2}}} u_{i_{1} \cdots i_{q}} s_{1} \wedge \cdots \wedge s_{n} \wedge \bar{s}_{i_{1}} \wedge \cdots \wedge \bar{s}_{q_{q}} .
\end{aligned}
$$

we have

$$
(\Theta \Lambda u, u)_{\Phi}=(\tilde{u}, \tilde{u})_{\Phi} \cdot
$$

On the other hand we have

$$
\begin{aligned}
& \exp (-\Phi) u \wedge \bar{*} f \\
& \quad=K \exp (\Phi) \sum_{i_{1}<\cdots<i_{q}} u_{i_{1} \cdots i_{q}} \overline{f_{i_{1} \cdots i_{q}}^{\prime}} s_{1} \wedge \cdots \wedge s_{n} \wedge \bar{s}_{1} \wedge \cdots \wedge s_{n}
\end{aligned}
$$

where

$$
f_{i_{1} \cdots i_{q}}^{\prime}=\frac{1}{\lambda_{1} \cdots \lambda_{n} \lambda_{i_{1}} \cdots \lambda_{i_{q}}} f_{i_{1} \cdots i_{q}} .
$$

By (29), (32) and (35) we have

$$
\begin{aligned}
& \left|u_{i_{1} \cdots i_{q}} \overline{f_{i_{1} \cdots i_{q}}^{\prime}}\right| \\
& =\frac{1}{\sqrt{\sum_{k=1}^{q} \lambda_{i_{k}}^{2}} \sqrt{\sum_{k=1}^{q} \lambda_{i_{k}}^{-2}}\left|\tilde{u}_{i_{1} \cdots i_{q}} \tilde{f}_{i_{1} \cdots i_{q}}\right|} \\
& \quad \leqq \frac{1}{q}\left|\tilde{u}_{i_{1} \cdots i_{q}} \tilde{f}_{i_{1} \cdots i_{q}}\right| .
\end{aligned}
$$

Therefore by Cauchy-Schwarz inequality we have

$$
\begin{aligned}
& \left|\int_{U} \exp (-\Phi) u \wedge \bar{*} f\right|^{2} \\
& \quad \leqq \frac{1}{q} \int_{U} \exp (-\Phi) \tilde{u} \wedge \bar{*} \tilde{\int} \int_{U} \exp (-\Phi) \tilde{f} \wedge \bar{*} \tilde{f}
\end{aligned}
$$

where $U$ is a sufficiently small neighbourhood of $x_{0}$. Since $x_{0}$ was arbitrary we obtain

$$
q\left|(u, f)_{\Phi}\right|^{2} \leqq(\tilde{u}, \tilde{u})_{\Phi}(\tilde{f}, \tilde{f})_{\Phi}=(\Theta \Lambda u, u)_{\Phi}\left(\Lambda^{-1} \Theta^{-1} f, f\right)_{\Phi} .
$$

Thus we obtain (20).

Q.E.D.

Combining Propositon 1.4 with a well known theorem (cf. e.g. [5, Theorem 1.1.4]) we obtain the following theorem (see also [6]). 
Theorem 1.5. Let $X, d s^{2}$ and $\Phi$ be as above. Let $f \in L_{2}^{n, q}(X), q \geqq 1$. Then there exists a $g \in L_{1}^{n, q-1}(X)$ satisfying $\bar{\partial} g=f$ if and only if $\bar{\partial} f=0$.

Since $L_{1}^{n, 0}(X)=L_{2}^{n, 0}(X)$, we have

Corollary 1.6. Let $X, d s^{2}$, and $\Phi$ be as above and let $f \in L_{2}^{n, 1}(X)$, then there exists a $g \in L_{2}^{n}, 0(X)$ such that $\bar{\partial} g=f$ if and only if $\bar{\partial} f=0$.

Corollary 1.7. Let $X$ be a complex manifold of dimension $n$ with a $C^{2}$ complete Kähler metric, and $\Phi$ a bounded strictly plurisubharmonic function on $X$. Assume that $\Phi$ is of class $C^{4}$. Then, for any $f \in L_{2}^{n, 1}(X)$ with $\bar{\partial} f=0$, there exists a $g \in L_{2}^{n}, 0(X)$ such that $f=\bar{\partial} g$.

Proof. Let $d s^{2}$ be the given complete Kähler metric on $X$. We set

$$
d \tilde{s}^{2}:=d s^{2}+d \sigma^{2} .
$$

Then the eigenvalues of $d \tilde{s}^{2}$ with respect to $d \sigma^{2}$ is larger than 1. Thus the hypothesis of Theorem 1.5 is satisfied.

\section{§ 2. Complete Kähler Domains with $\boldsymbol{C}^{1}$-Boundary}

Let $M$ be a complex manifold of dimension $n$. Let $X$ be a domain, i.e., a connected open set in $M . \quad X$ is called a domain with $C^{1}$-boundary if there exists a real valued continuously differentiable function $\rho$ defined on a neighbourhood $\tilde{V}$ of the boundary $\partial X$ of $X$ such that $\tilde{V} \cap X=\{x \in \tilde{V} ; \rho(x)<0\}$ and $\operatorname{grad} \rho$ is nonzero everywhere on $\partial X$. We call $\rho$ a defining function of $\partial X . X$ is called a complete Kähler domain if there exists a complete Kähler metric on $X$.

Example. Let $\mathbb{C}^{n}$ be the complex number space of dimension $n$. We let

$$
B(r)=\left\{\left(x_{1}, \ldots, x_{n}\right) \in \mathbb{C}^{n} ; \sum_{i=1}^{n}\left|x_{i}\right|^{2}<r\right\}, \quad r>0
$$

Then $B(r)$ is a complete Kähler domain with $C^{1}$-boundary (in $\mathbf{C}^{n}$ ). As a complete Kähler metric on $B(r)$ we may choose

$$
d s_{r}^{2}=\sum_{\alpha, \beta} \frac{\partial^{2} \eta(z)}{\partial z^{\alpha} \partial \bar{z}^{\beta}} d z^{\alpha} \cdot d \bar{z}^{\beta},
$$

where $z=\left(z^{1}, \ldots, z^{n}\right)$ and

$$
\eta(z)=\frac{1}{r-\sum_{\alpha=1}^{n}\left|z^{\alpha}\right|^{2}}
$$


More generally holomorphically convex domains in Stein manifolds are complete Kähler domains. The following lemma is obvious.

Lemma 2.1. Let $X_{1}$ and $X_{2}$ be complete Kähler domains with $C^{1-b o u n d a-~}$ ry in $M$. Then $X_{1} \cap X_{2}$ is a complete Kähler domain with $C^{1}$-boundary in $X_{1}$.

Definition 2.2. A domain $X$ in a complex manifold $M$ is called pseudoconvex if for every $x_{0} \in \partial X$ there exists a neighbourhood $U$ of $x_{0}$ in $M$ such that $U \cap X$ is a Stein manifold.

The following theorem is the main theorem of this section.

Theorem 2.3. Let $X$ be a complete Kähler domain with $C^{1}$-boundary in a complex manifold $M$. Then $X$ is pseudoconvex.

Proof. Let $X$ be a complete Kähler domain with $C^{1}$-boundary in $M$ and $x_{0} \in \partial X$. Let $W$ be a coordinate neighbourhood of $x_{0}$ with coordinate $\left(z^{1}, \ldots, z^{n}\right)$ such that $z^{i}$ are holomorphic on a neighbourhood of the closure of $W$ and

$$
W=\left\{x \in M ; \sum_{i=1}^{n}\left|z^{i}(x)\right|^{2}<1\right\} .
$$

After a linear change of coordinates we may assume that if we let $W_{\gamma}:=\{x \in W$; $\left.z^{n}(x)=\gamma\right\}(|\gamma|<1 / 2), X \cap W_{\gamma}$ is a domain with $C^{1}$-boundary in $W_{\gamma}$. By Lemma 2.1, $X \cap W$ and $X \cap W_{\gamma}$ are complete Kähler domains with $C^{1}$-boundary in $W$ and $W_{\gamma}$, respectively. We let

$$
B\left(x_{0}, r\right)=\left\{x \in W ; \sum_{i=1}^{n}\left|z^{i}(x)\right|^{2}<r\right\} \quad \text { for } \quad r<1 .
$$

Let $\rho$ be a defining function of $\partial X$. We choose $\left(\xi^{1}, \ldots, \xi^{n}, \eta^{1}, \ldots, \eta^{n}\right), \xi^{i}, \eta^{i} \in \mathbb{R}$, $i=1,2, \ldots, n$ satisfying

$$
\sum_{i=1}^{n}\left|\xi^{i}\right|^{2}+\sum_{i=1}^{n}\left|\eta^{i}\right|^{2}=1
$$

and

$$
\sum_{i=1}^{n} \frac{\partial \rho}{\partial u^{i}} \xi^{i}+\sum_{i=1}^{n} \frac{\partial \rho}{\partial v^{i}} \eta^{i}>0 \quad \text { at } \quad x_{0}
$$

where we set $z^{i}=u^{i}+\sqrt{-1} v^{i}$. Choosing $W$ smaller if necessary we may assume that $\rho$ is defined on $W$. For $r<1$ and $\varepsilon<1-r$ we define

$$
X_{r}^{\varepsilon}=\left\{x \in B\left(x_{0}, r\right) ; \rho(u(x)-\varepsilon \xi, v(x)-\varepsilon \eta)<0\right\},
$$

where $u(x)=u=\left(u^{1}, \ldots, u^{n}\right), v(x)=v=\left(v^{1}, \ldots, v^{n}\right)$ and so on. $X_{r}^{\varepsilon}$ is the domain 
in $B\left(x_{0}, r\right)$ obtained by shifting $\partial X$ by $\varepsilon$ in the direction of $(\xi, \eta)$. It is clear that for sufficiently small $r$ there exists $\varepsilon_{0}>0$ such that for any $\varepsilon<\varepsilon_{0}$ and $|\gamma|<r$, $X_{r}^{\varepsilon}$ and $X_{r}^{\varepsilon} \cap W_{\gamma}$ are complete Kähler domains with $C^{1}$-boundary in $B\left(x_{0}, r\right)$ and $B\left(x_{0}, r\right) \cap W_{\gamma}$, respectively. Moreover by (46) we may assume that for any $\varepsilon<\varepsilon_{0}$ the closures of $X \cap B\left(x_{0}, r\right)$ and $X \cap B\left(x_{0}, r\right) \cap W_{\gamma}$ in $B\left(x_{0}, r\right)$ are contained in $X_{r}^{\varepsilon}$ and $X_{r}^{\varepsilon} \cap W_{\gamma}$, respectively. We fix such $r$ and $\varepsilon_{0}$.

Now we prove the theorem by induction on the dimension $n$ of $X$. The case $n=1$ being well known we assume that $n \geqq 2$ and the theorem is valid for $k<n$. We are going to prove that $X \cap B\left(x_{0}, r / 2\right)$ is a Stein manifold. For this purpose it suffices to prove the following assertion (see [6] Theorem 2.6.5 and Theorem 5.4.6).

Assertion: There exists no $x_{1} \in \partial\left(X \cap B\left(x_{0}, r / 2\right)\right)$ satisfying the property that there is a neighbourhood $V$ of $x_{1}$ such that for any holomorphic function $f$ on $X \cap B\left(x_{0}, r / 2\right)$ there is a holomorphic function $\tilde{f}$ on $V$ which coincides with $f$ on $V \cap X \cap B\left(x_{0}, r / 2\right)$.

We prove this assertion by contradiction. Suppose that there exists such $x_{1} \in \partial\left(X \cap B\left(x_{0}, r / 2\right)\right)$ and $V$. Since $B\left(x_{0}, r / 2\right)$ is a Stein manifold $x_{1} \in \partial X \cap$ $B\left(x_{0}, r / 2\right)$. We fix $\gamma$ in such a way that $W_{\gamma} \cap V-X \neq \emptyset$. There exists $\varepsilon_{1}>0$ such that $\varepsilon_{0}>\varepsilon_{1}$ and $\partial\left(X_{r}^{\varepsilon_{1}} \cap W_{\gamma}\right) \cap V \neq \emptyset$. Since by the induction hypothesis $X_{r}^{\varepsilon_{1}} \cap W_{\gamma}$ is pseudoconvex in $B\left(x_{0}, r\right) \cap W_{\gamma}$, it follows that $X_{r}^{\varepsilon_{1}} \cap W_{\gamma}$ is Stein (see [6] Theorem 5.4.6). Hence there exists a holomorphic function $f$ on $X_{r}^{\varepsilon_{1}} \cap W_{\gamma}$ such that

$$
\sup _{x \in V \cap X_{r}^{\varepsilon_{1} \cap W_{\gamma}}}|f(x)|=\infty
$$

We define a holomorphic map $\pi: B\left(x_{0}, r / 2\right) \rightarrow W_{\gamma}$ by

$$
\pi\left(z^{1}, \ldots, z^{n}\right)=\left(z^{1}, \ldots, z^{n-1}, \gamma\right) .
$$

Since the closure of $X \cap B\left(x_{0}, r\right)$ in $B\left(x_{0}, r\right)$ is contained in $X_{r}^{\varepsilon_{1}}$, we can define a positive number $\delta_{0}$ as follows. If

$$
X \cap B\left(x_{0}, \frac{r}{2}\right) \subset \pi^{-1}\left(X_{r}^{\varepsilon_{1}} \cap W_{\gamma}\right)
$$

we let $\delta_{0}:=1$. Otherwise we let

$$
\delta_{0}:=\inf _{x \in X \cap B\left(x_{0}, r / 2\right)-\pi^{-1}\left(X_{r}^{\varepsilon} 1 \cap W_{\delta}\right)}\left|z^{n}(x)-\gamma\right|>0 .
$$

We fix $\delta>0$ so that 


$$
\delta<\delta_{0}
$$

Let $\chi$ be an infinitely differentiable function from $\mathbb{C}$ to $\mathbb{R}$ such that $\chi(w)=0$ if $|w| \geqq \delta, \chi(w)=1$ if $|w| \leqq \delta / 2$ and $0 \leqq \chi(w) \leqq 1$. We define a function $h$ on $X \cap$ $B\left(x_{0}, r / 2\right)$ by

$$
h(x)=\chi\left(z^{n}(x)-\gamma\right) \cdot f\left(\pi\left(z^{1}(x), \ldots, z^{n}(x)\right)\right) \quad \text { if } \quad\left|z^{n}(x)-\gamma\right|<\delta_{0}
$$

and

$$
h(x)=0 \quad \text { otherwise }
$$

Then

$$
\bar{\partial} h \wedge \frac{d z^{1} \wedge \cdots \wedge d z^{n}}{z^{n}-\gamma}
$$

is a $\bar{\partial}$ closed $(n, 1)$-form on $X \cap B\left(x_{0}, r / 2\right)$ which is square integrable with respect to the metric

$$
d \sigma^{2}=\sum_{\alpha, \beta} \frac{\partial^{2}\left(\sum_{i=1}^{n}\left|z^{i}\right|^{2}\right)}{\partial z^{\alpha} \partial \bar{z}^{\beta}} d z^{\alpha} \cdot d \bar{z}^{\beta} .
$$

Since $X \cap B\left(x_{0}, r / 2\right)$ is a relatively compact complete Kähler domain in $W$, by Corollary 1.7 there exists a square integrable $(n, 0)$ form $g$ on $X \cap B\left(x_{0}, r / 2\right)$ such that

$$
\bar{\partial} g=\bar{\partial} h \wedge \frac{d z^{1} \wedge \cdots \wedge d z^{n}}{z^{n}-\gamma}
$$

Hence letting $g=g^{1} d z^{1} \wedge \cdots \wedge d z^{n}$ we get

$$
\bar{\partial}\left(h-\left(z^{n}-\gamma\right) g^{1}\right)=0 .
$$

Therefore $h-\left(z^{n}-\gamma\right) g^{1}$ is a holomorphic function on $X \cap B\left(x_{0}, r / 2\right)$ and it coincides with $f$ on $W_{\gamma} \cap X \cap B\left(x_{0}, r / 2\right)$. Note that by the hypothesis $x_{1}$ is a point through which every holomorphic function on $X \cap B\left(x_{0}, r / 2\right)$ can be continued analytically to a holomorphic function on $V$. But this contradicts the fact that $h-\left(z^{n}-\gamma\right) g^{1}$ is holomorphic on $X \cap B\left(x_{0}, r / 2\right)$ and, by (48) combined with the theorem of identity, cannot be continued analytically across $\partial\left(X_{r}^{\varepsilon_{1}} \cap W_{\gamma}\right) \cap V$.

$$
\text { Q.E.D. }
$$

By the theorem of Docquier-Grauert [3] we have

Corollary 2.4. Let $M$ be a Stein manifold and $X$ be a domain with $C^{1-}$ boundary in $M$. Then $X$ is a Stein manifold if and only if $X$ has a complete Kähler metric. 
The corollary generalizes Satz $C$ of Grauert [4].

\section{References}

[1] Andreotti, A. and Vesentini, E., Carleman estimates for the Laplace-Beltrami equation on complex manifolds, Publ. Math., I.H.E.S., 25 (1965), 81-130.

[2] Calabi, E. and Vesentini, E., On compact locally symmetric Kähler manifolds, Ann. of Math., 71 (1960), 472-507.

[ 3 ] Docquier, F. and Grauert, H., Levisches Problem und Rungeschen Satz für Teilgebiete Steinscher Mannigfaltigkeiten, Math. Ann., 140 (1960), 94-123.

[4] Grauert, H., Charakterisierung der Holomorphiegebiete durch die vollständige Kählersche Metrik, Math. Ann., 131 (1956), 38-75.

[ 5 ] Hörmander, L., $L^{2}$ estimates and existence theorems for the $\bar{\partial}$ operator, Acta Math., 113 (1965), 89-152.

[6] - An Introduction to Complex Analysis in Several Variables, D. Van Nostrand, Princeton, N. J., 1966.

Added in proof: The author has obtained in a forthcoming paper 'Analyticity of complements of complete Kähler domains' that if $X$ is a domain in a complex manifold $M$ and $M-\mathrm{X}$ is a regular $C^{1}$-submanifold of real codimension 2, then $X$ is pseudoconvex (equivalently, $M-X$ is a complex submanifold of $M$ ). 